\title{
Suppression of 2D superconductivity by the magnetic field: quantum corrections vs superconductor-insulator transition.
}

\author{
V.F.Gantmakher, S.N.Ermolov, G.E.Tsydynzhapov $⿴$ and A.A.Zhukov \\ Institute of Solid State Physics RAS, Chernogolovka, Russia \\ T.I.Baturina \\ Institute for Semiconductor Physics SB RAS, Novosibirsk, Russia
}

\begin{abstract}
Magnetotransport of superconducting $\mathrm{Nd}_{2-x} \mathrm{Ce}_{x} \mathrm{CuO}_{4+y}(\mathrm{NdCeCuO})$ films is studied in the temperature interval $0.3-30 \mathrm{~K}$. The microscopic theory of the quantum corrections to conductivity, both in the Cooper and in the diffusion channels, qualitatively describes the main features of the experiment including the negative magnetoresistance in the high field limit. Comparison with the model of the field-induced superconductor-insulator transition is included and a crossover between these two theoretical approaches is discussed.
\end{abstract}

The superconductor-insulator transition (SIT) is an example of the quantum phase transitions [1] which constitutes drastic change of the ground state of the system at zero temperature with varying a parameter. The field was pioneered by A. Goldman et al. in the 1989 [2] who obtained the transition from insulating to superconductive state in the thin Bi film with the change of its thickness. Later, Fisher [3] suggested existence of magnetic field-induced SIT in two-dimensional (2D) systems and Hebard and Paalanen demonstrated [4, 5] such a transition in amorphous $\mathrm{InO}_{x}$ films. Numerous results obtained in several other materials by different groups $6,[7,[8,9]$ were also interpreted within the framework of the field-induced SIT. Main arguments in favor of this interpretation were negative derivative of resistance $\partial R / \partial T$ in the fields above the critical and existence of a finite-size scaling, i.e. existence of some critical region on the $(T, B)$-plane where the behavior of the system was governed by competition of the quantum phase transition correlation length $\xi \propto\left(B-B_{c}\right)^{-\nu}$ and thermal length $L_{T} \propto T^{1 / z}$ with $z$ and $\nu$ being constants called the critical exponents. All relevant quantities in this region are supposed to be universal functions $f$ of ratio of the lengths which can be written in the form of scaling variable $\left(B-B_{c}\right) / T^{1 / z \nu}$. For the resistivity in two dimensions $R_{\square}$ this dependence takes form [3]

$$
R_{\square}(B, T)=R_{c} f\left[\left(B-B_{c}\right) / T^{1 / z \nu}\right],
$$

where $R_{c}$ is a constant of the order of $h / 4 e^{2} \approx 6.5 \mathrm{k} \Omega$. It is called the critical resistance.

In the analysis of the experiments [6, 7, ,8, 9] the negative derivative $\partial R / \partial T$ was rated as an indicator of the insulating state. However, that is not enough: the characteristic of any insulator is the exponential temperature dependence of the resistance. This was demonstrated only in $\mathrm{InO}_{x}$ films [10]. The growth of the resistance with decreasing temperature on the non-superconducting side of the field-induced transition in the experiments with

*Electronic address: gombo@issp.ac.ru
MoGe [6], MoSi 7] and $\mathrm{NdCeCuO} \mathrm{8,} \mathrm{9]} \mathrm{was} \mathrm{minuscule,}$ about ten percent at its best. It reminded more a metal with quantum corrections to its conductivity than an insulator. Usually, the authors do not dwell on the issue, considering weak localization-like behavior to be telltale sign of insulator - as, according to scaling hypothesis [11], there is no non-superconducting delocalized state at zero temperature in $2 \mathrm{D}$ and weak localization is expected to transform sooner or later into strong. However, this crossover might be postponed to extremely low temperature which would never be achieved in practice.

There exists one more sign of SIT. According to the boson-vortex duality model [1, 3], the insulating state which appears as the result of SIT is rather specific; it contains pair correlations between the localized electrons as the remnant of the superconducting pairing. Such insulator is called the Bose-insulator [5] and the correlated electrons are called localized electron pairs. These correlations should be destroyed by strong magnetic field leading to increase of the carrier mobility, to the negative magnetoresistance 12] and even to a reentrant insulatornormal-metal transition [10]. The negative magnetoresistance was observed in $\mathrm{MoSi}$ [13] and $\mathrm{NdCeCuO} \mathrm{[9]}$. But it was much weaker than in InO, just the same as the growth of the resistance with decreasing temperature discussed above.

When comparing the whole set of data in $\mathrm{InO}[4,5,10$, 14] with those in MoGe [6], MoSi 7] and $\mathrm{NdCeCuO} \mathrm{[9]}$, one can't help impression that they have many similar features though of different scales of magnitude. At the same time, it was shown in a set of $\mathrm{InO}_{x}$ films with various oxygen content $x$ that in low-resistivity films a transition to the metallic state substitutes SIT, the rate of the temperature dependence scales down and the whole pattern of curves approaches that of the usual superconducting transition 10, 14]. The main idea of this paper follows from this observation. It is to compare experimental set of data of a "small-scale" type with the theory of the superconducting transition in dirty limit and, keeping in mind its features related to SIT, to build a bridge between SIT and thermodynamic superconducting transition. 
Experiment was performed on the $1000 \AA$-thick films of $\mathrm{Nd}_{2-x} \mathrm{Ce}_{x} \mathrm{CuO}_{4+y}(\mathrm{NdCeCuO})$ obtained by laser ablation with $\mathrm{CuO}_{2}$ planes parallel to the plane of the film. Films were not superconductive as-grown. In order to obtain superconductivity they were annealed at $720^{\circ} \mathrm{C}$ in the flowing ${ }^{4} \mathrm{He}$ gas for several hours. As we aimed to study vicinity of the SIT, we were not trying to reach maximal $T_{c}$ of this material, but were paying attention for smoothness and width of the zero-field transition. A sample was chosen with zero-field transition temperature $T_{c 0}=11.8 \pm 0.4 \mathrm{~K}$ (found by fitting of the superconducting fluctuation contribution to the conductivity above $\left.T_{c 0}\right)$ and the transition width $\Delta T \simeq 2 \mathrm{~K}$.

The resistivity was measured in the ab-plane by the 4-terminal technique. Both current and potential probes were attached on the surface of the films by silver paste. Distance between potential probes corresponded to one square. Magnetic field was applied perpendicular to the film plane (along c-axis). Data, both as a function of field at constant temperature and as a function of temperature at constant field, were obtained though only the latter will be presented below. The upper panel of Fig [1 presents an overview of the impact of the field on $R(T)$ dependence and the lower one zooms in on the region of interest, i.e. on the low temperature and high field region.

On the right axis of Fig. 11 the resistance reduced per one $\mathrm{CuO}_{2}$ plane per square is denoted. As $\mathrm{NdCeCuO}$ is highly anisotropic 15], it is reasonable to assume the film to be a stack of $2 \mathrm{D}$ conducting $\mathrm{CuO}_{2}$ planes with interplane spacing $6 \AA$, quasi-independent and connected in parallel. This is supported by observations of $2 \mathrm{D}$ character of quantum interference corrections [16] and magnetoresistance [17]. Later on we continue discussion in terms of this variable, disregarding full resistivity and actual thickness of the film. As one could see from Fig. [1] the value of the resistance per layer stays quite far from the quantum resistance $h / 4 e^{2}$ expected for the SIT.

The data are quite typical for the material (cf., for example, Ichikawa et al. [9]). In the low field region, the transition is shifted to the lower temperature as the field increases while the shape of the transition is preserved relatively well. Above $2 \mathrm{~T}$, the transition broadens drastically and eventually disappears; at about $3.5 \mathrm{~T}$, the $d R / d T$ changes its sign. At higher fields, above $5 \mathrm{~T}$, the resistance starts to decrease with the increasing field; it follows from the crossing of the $5 \mathrm{~T}$ and $7 \mathrm{~T}$ curves that a region of the negative magnetoresistance exists below $0.8 \mathrm{~K}$ and at $B>5 \mathrm{~T}$.

The set of curves $R(T)$ on the lower panel of Fig.1 is similar to those obtained in [6, 7, 8, [9] which had been regarded as a field-induced SIT. Low-field curves (which bend down) may be supposed to reach zero resistivity at zero temperature and to become superconductor, high field curves (which bend up) may be supposed to diverge toward zero temperature and become insulator. In between, there is a curve which is almost horizontal; it manifests itself as common crossing point

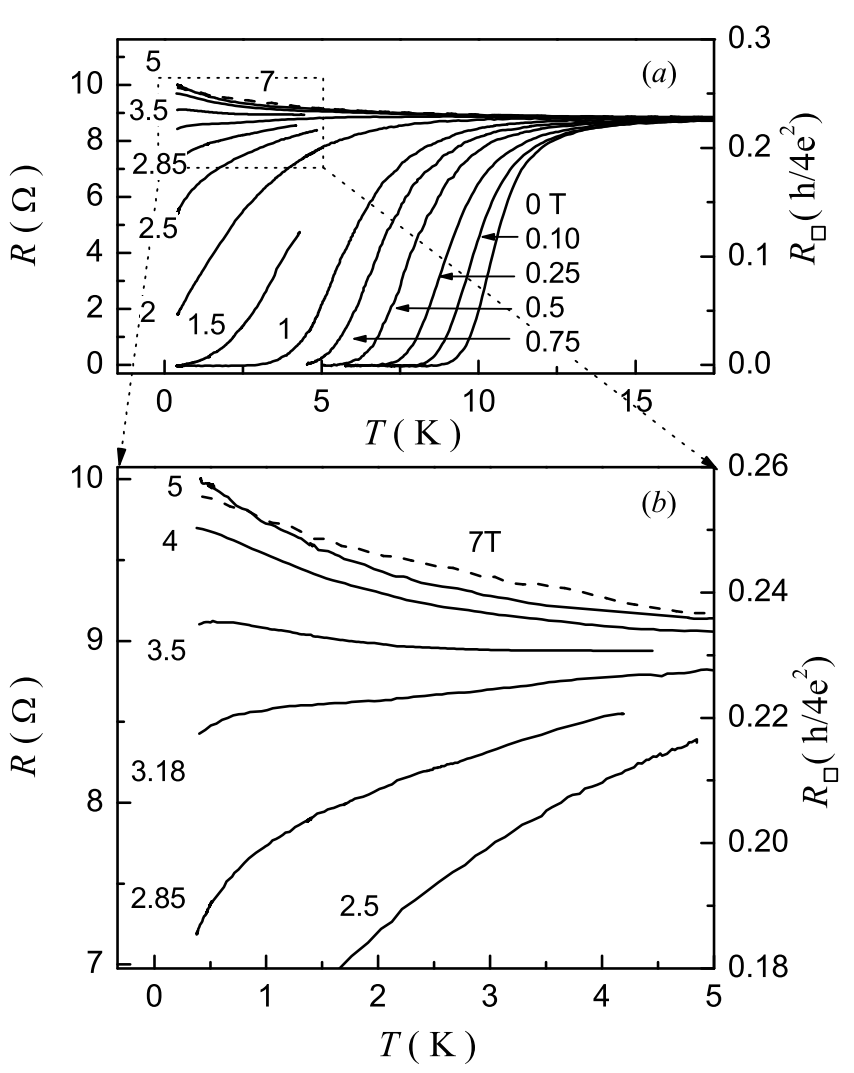

FIG. 1: Low-temperature resistivity data for the $\mathrm{NdCeCuO}$ film. Panel $b$ is an expansion of the designated area. Curve at $7 \mathrm{~T}$ (dash line) is crossing the other ones manifesting the negative magnetoresistance below $1 \mathrm{~K}$.

of all isotherms on the $R-B$ graph. The corresponding state should be considered as the critical one with the temperature-independent resistance at the critical field $B_{c} \approx 3.5 \mathrm{~T}$. But instead of seeking scaling parameters we shall compare experimental data with the microscopic theory of the superconducting transition in dirty limit formulated in terms of quantum corrections to the classical Drude conductivity $\sigma_{0}=e^{2} / h\left(k_{F} l\right)$ where $k_{F}$ is the Fermi wavevector and $l$ is the elastic mean free path. This comparison became executable due to recent progress in calculation of the corrections due to superconducting fluctuations [18].

All quantum corrections fall into two categories - a one-particle correction, usually called weak localization, and those due to e-e interactions. The latter are divided into a diffusion channel correction (also known as Aronov-Altshuler term) and Cooper channel corrections (also known as superconductive fluctuations corrections which include Aslamazov-Larkin, Maki-Thompson and DOS terms). Weak localization and Aronov-Altshuler corrections diverge at $T \rightarrow 0$, Cooper channel corrections diverge at $T \rightarrow T_{c}(B)$ with $T_{c}(B)$ being mean field transition temperature. When the superconductivity is suppressed by the magnetic field, $T_{c}(B) \rightarrow 0$ and all cor- 


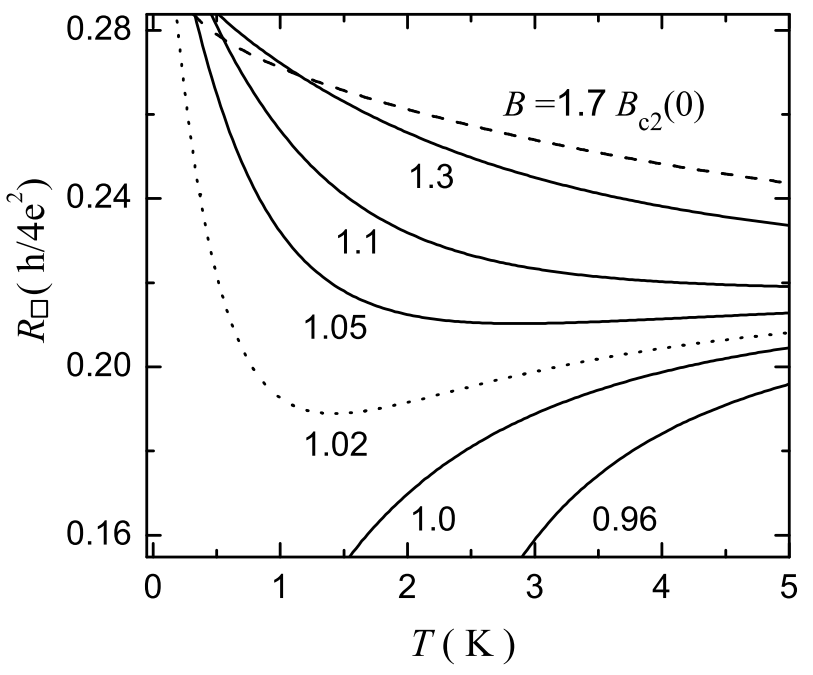

FIG. 2: Functions $R(T)$ at different $B$ calculated from Eqs. (2) and (3). The curves are labelled by reduced field values. The curve which shows the negative magnetoresistance is marked out by dash line. The dotted curve should not be compared to experiment, see text.

rections are important.

Recently, Galitski and Larkin [18] succeeded in extending calculations in the Cooper channel for twodimensional superconductors to the low temperature $T \ll T_{c}(0)$ and high magnetic field $B \gtrsim B_{c 2}(0)$. The correction to the conductivity in the dirty limit $\delta \sigma$ is obtained as the sum of contributions of ten Feynman diagrams in the first (one-loop) approximation and can be written in the form

$$
\delta \sigma=\frac{4 e^{2}}{3 \pi h}\left[-\ln \frac{r}{b}-\frac{3}{2 r}+\psi(r)+4\left(r \psi^{\prime}(r)-1\right)\right],
$$

where $r=\left(1 / 2 \gamma^{\prime}\right)(b / t), \gamma^{\prime}=e^{\gamma}=1.781$ is the exponential of Euler's constant, and $t=T / T_{c 0} \ll 1$ and $b=\left(B-B_{c 2}(T)\right) / B_{c 2}(0) \ll 1$ are reduced temperature and magnetic field.

To compare these calculations with the experiment, we added to the correction (2) an additional term to account for Aronov-Altshuler contribution, which is assumed to be field independent. Weak localization was omitted because we are interested in the region of rather strong magnetic fields where this correction was expected to vanish. Finally, we arrived at the formula

$$
R_{\square}^{-1}(B, T)=\sigma_{0}+\delta \sigma(B, T)-\alpha \frac{e^{2}}{h} \ln \left(T / T^{*}\right) .
$$

Inserting $T_{c 0}=11.8 \mathrm{~K}$ and experimental value of the classical conductivity $\sigma_{0}=1 / R_{\square}(7 \mathrm{~T}, 20 \mathrm{~K})$, and choosing $T^{*}=20 \mathrm{~K}$ to make the last term zero at $20 \mathrm{~K}$ and $\alpha=1 / 2$ to match the temperature dependence of the experimental curve at $7 \mathrm{~T}$, we get the plot of Fig. 2 which can be compared with the experimental one, Fig.11 . (Note, that

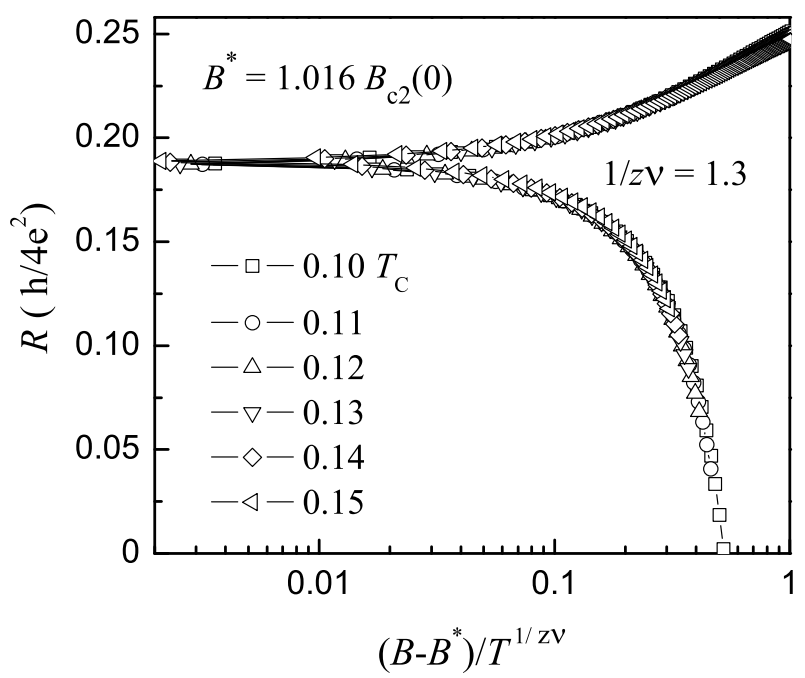

FIG. 3: "Scaling" of the curves calculated from Eqs. (2) and (3) in the same way as Fig 2 Restricted ranges of $T$ and $B$ are selected, see text.

on Fig.2] curves are labelled by reduced field values, those in units of $B_{c 2}(0)$. The same can't be done on Fig. [1 because experimental value of $B_{c 2}(0)$ is a bit uncertain.)

As one can see, the picture bears clear resemblance to the experiment - there is separation between low-field curves which "bend down", and high-field which "bend up"; there is also high field negative magnetoresistance at low temperature. There are two remarkable points: i) the scales of variation of resistance both with temperature and magnetic field are correct; ii) the region and the magnitude of the negative magnetoresistance are in reasonable agreement with the experiment as well.

However, the similarity is qualitative. It is difficult to make it quantitative and both the experiment and the theory are responsible for this.

Disadvantage of the experiment is hidden in macroinhomogeneity of the film. It follows from Fig. 2 that small $2 \%-3 \%$ changes of $B_{c 2}(0)$ lead to drastic shift in the shape of curves $R(T)$, especially near the critical value of $B$. Inevitable dispersion of the values of $B_{c 2}(0)$ along the film smoothes the curves and clears away the extremum. Hence, one should scarcely expect to find in the experimental assortment of curves one similar to the theoretical curve labelled 1.02 (plotted by the dotted line on Fig.22).

The expression (2) is apparently very sensitive to the function $B_{c 2}(T)$. Basically, this function is an implicit parameter of the theory. In Ref.18, authors used for $B_{c 2}(T)$ the mean-field function from the WerthamerHelfand-Hohenberg theory. It is doubtful, that this theory is applicable to high-resistive $2 \mathrm{D}$ objects, especially, as the shape of transition in 2D case should be affected by the vortex motion (Berezinsky-Kosterlitz-Thouless theory).

As a side note, a comment about the finite-size scal- 
ing equation (11) related to SIT. Certainly, expression (3) does not have form of equation (1) and no genuine scaling exists. However, in a restricted region of values of $T$ and $B$ representation of the theoretical curves in the form (11) can be done. This is illustrated by Fig. 3 where calculated data from the region $0.98<B / B_{c 2}(0)<1.2$ and $0.1<T / T_{c}<0.15$ are used for the tracing. As the "critical" magnetic field $B^{*}=1.016 B_{c 2}(0)$, the crossing point of several isotherms $R(B)$ was taken; $B^{*}$ is the field where the minimum of the isomagnetic curve $R(T)$ is located in the middle of the chosen temperature region. (Actually, in the limited range of parameters $B$ and $T$ scaling always exists provided that several curves $R(B)$ have a common crossing point.) It follows that the scaling tracing is necessary but not sufficient element of the analysis of the SIT, especially taking into account that we always deal with the limited temperature range in the experiment.

Appearance of the negative correction to conductance in the microscopic theory of the superconductive fluctuations 18 is very remarkable. It confirms that the superconducting correlations may lead at fields above the critical one not to the drop but to upsurge of the resistance. This can be regarded as the tendency toward the Bose-insulator, which could be distinguished from the Aronov-Altshuler term because it leads to the negative magnetoresistance. All the materials mentioned above can be lined up demonstrating continuous crossover from the Bose-insulator and gigantic negative magnetoresistance in InO to faint low-temperature uprise of the resistance and its tiny drop in strong magnetic fields in MoSi and $\mathrm{NdCeCuO}$. In essence, these films are similar to each other: they are uniform, highly disordered films, with the resistance close to quantum value $h / 4 e^{2}$. Nevertheless, experimental observations on $\mathrm{InO}_{x}$ and, for example, on $\mathrm{NdCeCuO}$ are quite different and there is a reason for it.

There is little doubt that at low enough temperature the growth of $R(T)$ we observe in the high magnetic field, i.e. in the normal state, will turn exponential. According to phenomenological estimate suggested by Larkin and Khmel'nitskii [19], the crossover happens when the corrections to the conductivity reach the level of the conductivity itself. The condition $\sigma_{0} \sim\left(e^{2} / h\right) \ln T$ gives crossover temperature

$$
T_{L K h} \simeq \frac{\varepsilon_{F}}{k_{F} l} e^{-2\left(k_{F} l\right)},
$$

where $\varepsilon_{F}$ and $k_{F}$ are the Fermi energy and the Fermi wavevector and $l$ is the elastic mean free path 19. Below this temperature there will definitely be a superconductive state at low field and pronounce insulating behavior at high field and there would be clear reason to apply SIT framework. So, the quantum corrections to the conductivity and the quantum phase transition phenomena are manifested at different temperature regions.

Though $T_{L K h}$ may be very low for normal metal $\left(T_{L K h} \lesssim 1 \mathrm{mK}\right.$ already for $\left.k_{F} l \approx 5\right)$, there are clear experimental indications that crossover to bosonic insulator behavior (that is, to the SIT framework) in the

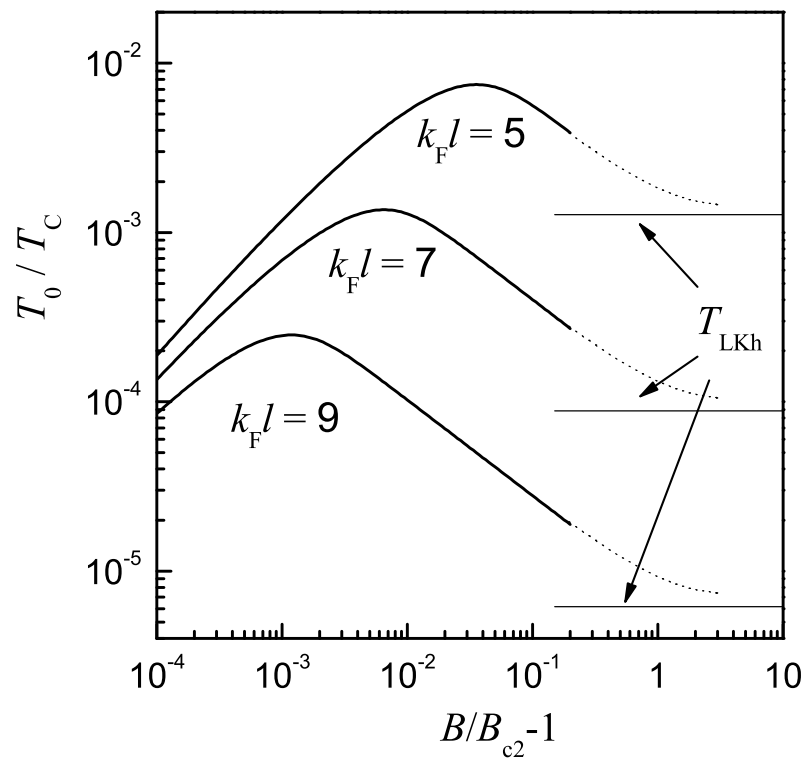

FIG. 4: Crossover temperature $T_{0}$ for several reduced values of the mean free path $l$ calculated by equating to zero the right part of the Eq. (3) for the fields values up to $B=1.2 B_{c 2}(0)$. Dotted lines qualitatively designate the asymptotic parts of the curves. Levels of $T_{L K h}$ approximately corresponding to the same values of $l$ are marked by horizontal lines.

intermediate field range, where pair correlations are still important, occurs at higher temperature [10]. This is consistent with theoretical observation [20] that the attractive interaction stimulates localization by combining single particles into pairs.

By equating two last terms in the relation (3) to the $\sigma_{0}$ and solving ensued equation one gets crossover temperature to bosonic insulator $T_{0}$ as the function of the magnetic field. These curves for $\sigma_{0}$ equal $5 e^{2} / h$ (or $\left.k_{F} l=5\right), 7 e^{2} / h$ and $9 e^{2} / h$ are presented on Fig 4 by solid lines. Thin solid lines present levels of $T_{L K h}$ determined by using only last term in the relation (3) and corresponding $\sigma_{0}$. As the equation (2) is valid only in the fields close to $B_{c 2}(0)$, the parts of curves in the higher fields, where $T_{0}(B)$ approaches $T_{L K h}$, are indicated qualitatively by dotted lines. In agreement with Refs. 10, 20, the crossover to activation behavior in the medium-range fields occurs at the temperatures more than order of magnitude higher than $T_{L K h}$. At the same time, the crossover temperature falls off exponentially with increasing classical conductivity so that for the actual value of our experiment it becomes infinitesimal. That's why field-induced SIT is so manifest in the $\mathrm{InO}_{x}$, whereas it is not observed in $\mathrm{MoGe}$ or $\mathrm{NdCeCuO}$, and there is no slightest sign of it in the $\mathrm{Al}$ film (note that according to the scaling hypothesis 11] any metal film should become insulating at $T=0$ if the superconductivity is destroyed by the magnetic field).

To summarize, we compared experimental data obtained on two-dimensional $\mathrm{NdCeCuO}$ superconductor in magnetic field at low temperature with the calculations 
of quantum corrections to the conductivity and found reasonable agreement. Lack of the activation behavior at high fields (on the "insulating side of transition") was the main reason which made inferior comparison of the same data with the model of field-induced SIT. Apparently, this happened because the temperature range turned out to be too high for this specific material. The type of the resistance dependence on the temperature is the guide in choosing the theoretical approach. To employ framework of the SIT in its full for $\mathrm{NdCeCuO}$, further essential lowering of the temperature is necessary.

This work was supported by grants RFBR-02-0216782, RFBR-02-02-08004, RFBR-03-02-16368 and by grant of Ministry of Science.
[1] S.L.Sondhi, S.M.Girvin, J.P.Carini, and D.Shahar, Rev. Mod. Phys 69, 315 (1997)

[2] D.B. Haviland, Y.Liu, and A.M. Goldman, Phys. Rev. Lett. 62, 2180 (1989).

[3] M.P.A. Fisher, Phys. Rev. Lett. 65, 923 (1990).

[4] A.F.Hebard and M.A. Paalanen, Phys. Rev. Lett. 65, 927 (1990).

[5] M.A. Paalanen, A.F. Hebard, and R.R. Ruel, Phys. Rev. Lett. 69, 1604 (1992).

[6] A. Yazdani and A. Kapitulnik, Phys. Rev. Lett. 74, 3037 (1995).

[7] S.Okuma, T.Terashima, and N.Kokubo, Solid State Comm. 106, 529 (1998).

[8] S.Tanda, S.Ohzeki, and T.Nakayama Phys. Rev. Lett. 69, 533 (1992).

[9] F.Ichikawa, Y.Yamasaki, T.Nishizaki, T.Fukami, T.Aomine, S.Kubo, and M.Suzuki, Solid State Comm. 98139 (1996).

[10] V.F. Gantmakher, M.V. Golubkov, V.T. Dolgopolov, G.E. Tsydynzhapov, and A.A.Shashkin, Pis'ma v ZhETF 68, 337 (1998).

[11] E. Abrahams, P.W. Anderson, D.C. Licciardello, and
T.V. Ramakrishnan, Phys. Rev. Lett. 42, 673 (1979).

[12] J. Lages and D.L. Shepelyansky, Phys. Rev. B 64, 094502 (2001)

[13] S.Okuma, T.Terashima, and N.Kokubo, Phys. Rev. B 58, 2816 (1998).

[14] V.F. Gantmakher, M.V. Golubkov, V.T. Dolgopolov, G.E. Tsydynzhapov, and A.A.Shashkin, Pis'ma v ZhETF 71, 231, 693 (2000).

[15] T.B. Charikova, A.I. Ponomarev, A.N. Ignatenkov et al. Fizika Metallov i Metallovedenie 91(2), 46 (2001).

[16] G.I. Harus, A.I. Ignatenkov, A.I. Ponomarev et al. Pis'ma v ZhETF 70, 93 (1999).

[17] A. Kussmaul, J.S. Moodera, P.M. Tedrow et al. Physica C 177, 415 (1991).

[18] V.M. Galitski and A.I. Larkin, Phys. Rev. B 63, 174506 (2001).

[19] A.I. Larkin and D.E. Khmel'nitskii, ZhETF 83, 1140 (1982)

[20] B. Srinivasan, G. Benenti, and D.L. Shepelyansky, Phys. Rev. B 66, 172506 (2002) 